\title{
Profiles of Cognitive Dysfunction in Chronic Amphetamine and Heroin Abusers
}

T. J. Ornstein, B.A., J. L. Iddon, Ph.D., A. M. Baldacchino, M.D., MRCPsych., B. J. Sahakian, Ph.D., M. London, MBChB, MRCPsych., B. J. Everitt, Ph.D., and T. W. Robbins, Ph.D.

Groups of subjects whose primary drug of abuse was amphetamine or heroin were compared, together with ageand IQ-matched control subjects. The study consisted of a neuropsychological test battery which included both conventional tests and also computerised tests of recognition memory, spatial working memory, planning, sequence generation, visual discrimination learning, and attentional set-shifting. Many of these tests have previously been shown to be sensitive to cortical damage (including selective lesions of the temporal or frontal lobes) and to cognitive deficits in dementia, basal ganglia disease, and neuropsychiatric disorder. Qualitative differences, as well as some commonalities, were found in the profile of cognitive impairment between the two groups. The chronic amphetamine abusers were significantly impaired in performance on the extra-dimensional shift task (a core component of the Wisconsin Card Sort Test) whereas in contrast, the heroin abusers were impaired in learning the normally easier intra-dimensional shift component. Both groups were impaired in some of tests of spatial working memory. However, the amphetamine group, unlike the heroin group, were not deficient in an index of strategic performance on this test. The heroin group failed to show significant improvement between two blocks of a sequence generation task after training and additionally exhibited more perseverative behavior on this task. The two groups were profoundly, but equivalently impaired on a test of pattern recognition memory sensitive to temporal lobe dysfunction. These results indicate that chronic drug use may lead to distinct patterns of cognitive impairment that may be associated with dysfunction of different components of cortico-striatal circuitry.

[Neuropsychopharmacology 23:113-126, 2000] (C) 2000 American College of Neuropsychopharmacology. Published by Elsevier Science Inc. All rights reserved
KEY WORDS: Drug abuse; Cognition; Amphetamine; Stimulant; Heroin; Prefrontal cortex

Chronic misuse of stimulant drugs, such as cocaine or amphetamine, or of opiates such as heroin may lead to long-lasting impairments in brain function (e.g., Ricaurte et al. 1984: Miller 1985). Such effects potentially add to

From the Department of Psychiatry, University of Cambridge School of Clinical Medicine, Addenbrookes Hospital, Cambridge, UK (TJO, JLI, BJS); Department of Experimental Psychology, University of Cambridge, Cambridge, UK (JLI, BJE, TWR); and Drug and Alcohol Service, Brookfields Hospital, Cambridge, UK (AMB, ML).

Address correspondence to: Dr. T.W. Robbins, Department of Experimental Psychology, University of Cambridge, Downing Street, Cambridge, CB2 3EB, UK.

Received June 24, 1999; revised November 23, 1999; accepted February 1,2000 . the burden of treatment in humans, either by requiring additional rehabilitation for cognitive deficits that impair everyday function, or else by strengthening the drug-seeking urge through ancillary effects on behavior. However, there have been relatively few detailed investigations of the nature of the possible neuropsychological changes associated with long-term stimulant or opiate abuse, particularly when comparing profiles of impairment in the same study.

Heavier use of opiates in long-term users has been shown to be associated with greater likelihood of neuropsychological impairment as assessed by a battery including the WAIS, aphasia tests, and the Halstead battery (Grant et al. 1978). Hill and Mikhael (1979) who studied opiate abusers with an almost exclusive drug preference for heroin, found that they were impaired on 
Tactual Performance for memory and location and Tapping Tests, but not on the Category Test, a measure of abstract reasoning ability. They concluded that, since performance on the Category Test is thought to be related to damage to the frontal lobes, this brain region may be less affected by opiate abuse. This conclusion is supported to some extent by results from studies that have failed to detect a difference between opiate users and controls on other measures of neuropsychological functioning thought to correlate with frontal lobe damage; for example, abstract thinking (Bruhn and Maage 1975), or verbal fluency (Rounsaville 1982).

Several studies have assessed cognitive function in stimulant (cocaine) abusers (Washton and Gold 1984; Ardila et al. 1991; Mittenberg and Motta 1993). In an extensive study of 61 cocaine abusers and 59 polyabusers, Rosselli and Ardila (1996) found significant impairments in short-term memory and attention. Abstracting ability as tested by the Wisconsin Card Sorting Test and non-verbal short-term memory, were less impaired, while verbal fluency and long-term memory were unimpaired. Test scores were found to correlate with lifetime cocaine abuse, suggesting a relationship between drug abuse and cognitive dysfunction. Similar patterns of impairment have been reported by others (Parsons and Farr 1981; Washton and Gold 1984; Carlin and O'Malley 1996). O'Malley et al. (1992) found mild but significant impairments in tests of attention and memory in 20 heavy cocaine abusers. These subjects also performed poorly on the Category Test, but surprisingly were superior in verbal fluency tests.

The cortical distributions of dopaminergic and opiate receptors (Joyce and Meador-Woodruff 1997; Mansour et al. 1988) might be expected to lead to different patterns of cognitive impairment among stimulant and opiate abusers. For example, dopamine (DA) D1 receptors are mainly present in the anterior neocortex (especially prefrontal cortex). Subcortically, stimulants and opiates have distinct effects in the nucleus accumbens, but share some common actions, for example, in boosting the activity of the mesolimbic dopamine system (Koob and LeMoal 1997; Wise and Bozarth 1984). Consequently, some similarities in the profiles of neuropsychological impairment might also result from long-term abuse of these drugs. Chronic abuse of stimulants, and also opiates, may lead to changes in neurotransmission present in DA terminals such as the nucleus accumbens, caudate-putamen, and frontal cortex (Kalivas and Sorg 1997), leading to disruptive functioning of corticostriatal loops subserving cognitive and affective information processing (Alexander et al. 1986).

A variety of cognitive impairments has been reported in patients with frontal-striatal pathology which are often subsumed under the rubric of 'executive dysfunction.' Patients with frontal lobe lesions present with cognitive function deficits in tests of working memory, attentional set-shifting, planning ability and strategic learning (Shallice 1982; Owen et al. 1995a, 1990, 1991; Milner 1963). Similarly, patients with Parkinson disease exhibit deficits in tests of 'frontal function' (Downes et al. 1989; Owen et al. 1992, 1993), which are exaggerated by L-Dopa withdrawal, suggesting an involvement of brain dopamine systems (Lange et al. 1992; Owen et al. 1995a). Currently, there is considerable debate as to the extent to which such executive functions might be dissociable and subject to differential modulation by ascending neurotransmitter systems (Robbins 1996).

The present study was designed to further define and compare the patterns of cognitive impairment among chronic amphetamine and heroin abusers and to relate neuropsychological dysfunction to clinical variables. Tests shown to be sensitive to fronto-striatal or temporal lobe damage were employed from a standardized and computerised neuropsychological test battery (CANTAB) (Robbins et al. 1994, 1998) together with other related variants (Iddon et al. 1998; Owen et al. 1995a). The CANTAB battery has been used in a wide variety of relevant patient groups, including patients with neurosurgical excisions of the prefrontal cortex or temporal lobe (Owen et al. 1995b), basal ganglia disorders such as Parkinson's (e.g., Downes et al. 1989; Owen et al. 1992), and Huntington's disease (e.g., Lawrence et al. 1996), as well as depression, schizophrenia (Elliott et al. 1998), and dementia (Sahakian et al. 1988). The battery has also been previously employed in psychopharmacological (Lange et al. 1992; Elliott et al. 1997; Robbins et al. 1998) and toxicological (Maruff et al. 1998) investigations. Also, most importantly, tests from the battery have been used in the context of functional neuroimaging (Baker et al. 1996; Owen et al. 1996a; Rogers et al. 1999a) which may be especially useful eventually for providing a neurocognitive profile of the effects of long-term opiate or amphetamine abuse.

Substance misusers are a heterogeneous group and primary users of one class of drugs will inevitably at some point in time have used drugs of another class. However, within this spectrum of use one can separate out groups on the basis of personal preference and relative frequency and duration of use. This method has been used in epidemiological research on amphetamine users in Cambridge (Haarhoff and London 1995).

In this study, we compared the cognitive profiles of two separate groups of chronic drug abusers who met the DSM-IV criteria for chronic heroin or chronic amphetamine dependence. Within the chronic amphetamine abuse group, was a subgroup of subjects who also met the criteria for opiate dependence (though with a significantly shorter duration of opiate abuse than amphetamine abuse), and were thus distinct from the subgroup of chronic amphetamine abusers who were not opiate dependent. Therefore, an additional 
analysis directly compared the cognitive performance of these two subgroups.

\section{METHODS}

\section{Subjects}

Heroin Abusers. Twenty-two subjects (all males) were recruited through the Drug and Alcohol Dependency Unit at Brookfields Hospital, Cambridge. They had all used opiates regularly for a minimum of three years and all met the APA Diagnostic and Statistical Manual of Mental Disorders - 4th edition (DSM-IV) (Task Force of American Psychiatric Association 1994) criteria for Opiate (Physiological) Dependence, but not Amphetamine (Physiological) Dependence. This required the presence in the preceding twelve months of either tolerance or withdrawal symptoms and at least two of the following features: opiates taken in larger quantities or longer than intended, craving or failed efforts to cut down, drug directed activity, salience of drug related behaviour, and persistent use despite awareness of problems.

Drug histories were taken prior to testing [by a consultant psychiatrist (ML) in conjunction with another psychiatrist $(\mathrm{AB})$ ]. The mean age of this group was 33.3, $\mathrm{SD} \pm 7.8$ (range 22 to 50 ) years and their promorbid verbal IQ as estimated using the National Adult Reading Test (NART) (Nelson 1982) was 108.9, SD \pm 9.4 (range 93 to 123). The mean duration of heroin abuse was 11.61, SD \pm 8.3 (range 3 to 30 ) years. Educational attainment was measured by last year of education. Three subjects had no further education beyond the age of 14, whereas two subjects proceeded beyond the age of 19. At the time of testing, 20 subjects were taking regular prescriptions of methadone, two naltrexone, and one carbamazepine. Two subjects reported drinking more than 30 units of alcohol (normal levels being 20 units for men and 14 for women) and three subjects described amphetamine use, but did not meet criteria for dependence. Two subjects used ecstasy (MDMA) irregularly and five took benzodiazepines intermittently. Almost all the heroin abusers $(21 / 22)$ reported regular cannabis use.

Amphetamine Abusers. Twenty-three subjects (19 male and four female) were recruited from the Drug and Alcohol Dependency Unit, as described above. All subjects had used amphetamines regularly for a minimum of three years and met the DSM-IV criteria for Amphetamine (Physiological) Dependence (similar criteria to those described above for opiate dependence). All were under the care of ML. The ages of the participants ranged from 18 to 51 with a mean age of $34.6, \mathrm{SD} \pm 9.2$ years. Verbal premorbid IQ as estimated using the NART was $108.7 \pm 7.0$ (range 92 to 126 ). One subject had not progressed educationally beyond 14 years, whereas five subjects continued after age 19. The mean duration of amphetamine abuse was $13.04(\mathrm{SD} \pm 7.0)$ years with a range of 5 to 29 years.

A total of 10 subjects of the 23 were receiving prescribed oral dexamphetamine and 13 were taking illicit amphetamine. At the time of testing, nine of the 23 subjects met the DSM-IV criteria for opiate dependence and were also receiving prescribed oral methadone (amphetamine + opiate subgroup). This subgroup had abused amphetamine for a mean of 18.44 (8.93) years and opiates for 12.28 (7.52) years. In every case, duration of amphetamine abuse had been longer than for opiates. Five of the methadone prescribed subjects were also receiving prescribed amphetamine. The remaining four methadone treated subjects had received a prescription of methadone for less than six months (while their duration of stimulant use had exceeded five years). The remaining 14 subjects were termed the amphetamine only sub-group, their duration of amphetamine abuse averaging 11.21 (6.92) years.

From the overall group of 23 subjects, one subject reported drinking more than 56 units per week and two gave a history of past heavy drinking. Subjects also reported intermittent abuse of cannabis $(n=20)$, MDMA $(n=9)$, and benzodiazepines $(n=5)$.

All subjects spoke English as their primary language. Exclusion criteria for both the heroin and amphetamine abusers included a history of psychiatric or neurological illness, clinical depression, and alcohol dependence. A minimum drug dependence duration of three years with major drug of abuse being either amphetamine or opiate was required for inclusion within the study. At the time of testing, no subjects were experiencing withdrawal symptoms or clinical features of intoxication.

Control Subjects. Normal healthy subjects were chosen to match the two drug abuse groups as closely as possible for age and premorbid verbal IQ as estimated using the NART (Nelson 1982). Control subjects were drawn from a pool of 48 volunteers recruited through advertisements placed in a Cambridge employment centre. The same subjects (control group $1 ; n=22$ ) were selected for each of the tests of verbal fluency, pattern and spatial recognition memory, spatial working memory, and one-touch Tower of London. For the attentional set-shifting task and the visuospatial strategy task, separate subgroups (control groups 2 and 3, respectively; each $n=22$ ) were formed. Control group 1 contributed three subjects to group 2 and 15 to group 3 . There was no overlap between groups 2 and 3 . The study was approved by the Cambridge Local Research Ethics Committee and all subjects gave informed consent for participation in the study.

A summary of characteristics for the two drug abuse groups and the control subjects is shown in Table 1. One-way analyses of variance revealed that both the 
Table 1. Subject Characteristics

\begin{tabular}{|c|c|c|c|c|c|c|c|}
\hline Group & Tests & $\mathbf{N}$ & Age & $\begin{array}{l}\text { Sex } \\
M: F\end{array}$ & $\begin{array}{c}\text { VIQ } \\
\text { (NART) }\end{array}$ & $\begin{array}{c}\text { Education } \\
\text { (School Leaving Age) }\end{array}$ & $\begin{array}{c}\text { Drug Abuse } \\
\text { Duration (Years) }\end{array}$ \\
\hline Heroin abusers & & 22 & $33.3(7.8)$ & $22: 0$ & $108.9(9.4)$ & $15.9(2.6)$ & $11.6(8.3)$ \\
\hline Amphetamine abusers & & 23 & $34.6(9.2)$ & $19: 4$ & $108.7(7.0)$ & $16.7(2.1)$ & $13.0(7.0)$ \\
\hline Control group 1 & $\begin{array}{l}\text { Verbal fluency } \\
\text { Pattern and spatial recognition } \\
\text { Spatial working memory } \\
\text { one-touch Tower of London }\end{array}$ & 22 & $31.6(8.0)$ & $10: 12$ & $108.9(9.0)$ & $18.2(1.6)$ & \\
\hline Control group 2 & Attentional set-shifting task & 22 & $33.1(13.5)$ & $15: 7$ & $112.8(8.5)$ & $18.1(2.7)$ & \\
\hline Control group 3 & Visuospatial Strategy task & 22 & $33.1(9.5)$ & $10: 12$ & $109.1(8.7)$ & $18.3(2.3)$ & \\
\hline
\end{tabular}

Standard deviation in brackets.

heroin and amphetamine abuse groups were well matched with their respective control subjects for selective tests; in terms of both age [verbal fluency, pattern and spatial recognition, spatial working memory, onetouch Tower of London: $\mathrm{F}(2,64)=0.75$, n.s.; attentional set-shifting: $F(2,64)=0.14$, n.s.; visuospatial strategy task: $\mathrm{F}(2,64)=0.19$, n.s.] and premorbid verbal IQ $[F(2,64)=0.01$, n.s.; $F(2,64)=1.51$; n.s.; $F(2,64)=$ 0.01, n.s.].

\section{Procedures}

Verbal Fluency. The verbal fluency test sensitive to frontal lobe function (Benton 1968) was administered using both letter and semantic categories. In the letter fluency subtest, subjects were asked to generate as many words as possible in a minute starting with the letter ' $\mathrm{F}$ '. The subjects then repeated this task with the letters ' $\mathrm{A}$ ' and ' $S$ '. In the category fluency subtest, they were asked to generate as many names from the semantic category 'animals' within 90 seconds. Mean word generation for both letter and category fluency was measured.

Computerised Tests. Some of the main tests were administered from the CANTAB battery (CeNeS Ltd. Cambridge, U.K.), a series of computerised tasks run on a portable microcomputer with a Datalux touch sensitive screen. Subjects were seated approximately $0.5 \mathrm{~m}$ from the touchscreen and were introduced to the apparatus through a 'motor screening task.' The subjects responded to 10 flashing red and green crosses presented in varying locations on the screen by touching the center of each cross with the index finger of their preferred hand. After completion of this task, the subjects were given the following tasks in the order described below.

Pattern and Spatial Recognition. These tests assess visual pattern and visuospatial recognition memory (Sahakian et al. 1988). In the pattern recognition task, which is more sensitive to temporal than frontal lobe dysfunction (Owen et al. 1995b), subjects were presented with a successive series of twelve colored ab- stract patterns. After a delay, the twelve patterns reappeared in reverse order alongside a novel pattern and they identified which of the two simultaneous presented patterns they had seen before. This procedure was then repeated with a new set of twelve patterns.

In the spatial recognition task, which is more sensitive to frontal lobe rather than temporal lobe dysfunction (Owen et al. 1995b), five squares were presented sequentially in different locations around the screen. Subjects were presented with a choice of two squares in different locations, one of which was novel; they had to touch the location in which the square had appeared previously. This procedure was repeated a further three times. Performance was measured by the number of correct responses calculated from 24 trials for pattern recognition and 20 trials for spatial recognition. Mean response latencies for both tasks were also measured.

Attentional Set-Shifting Task. A widely used assessment of cognitive set-shifting that requires shift of attention set from one perceptual category to another (e.g., shape to number) and which is attributed to prefrontal cortex integrity, is the Wisconsin Card Sorting Test (WCST) (Grant and Berg 1948). Modelled after but unlike the WCST, the CANTAB attentional set-shifting task (ID/ED) (Downes et al. 1989) was designed to assess the contribution of component processes to impaired set-shifting ability. The ID/ED task requires subjects to learn a series of two-alternative forced choice discriminations and is composed of nine stages beginning with a simple discrimination and its reversal of one dimension (e.g., two different lines). Compound discrimination and its reversal are then tested with the addition of other dimensions (e.g., two purple filled shapes) overlapping the lines. Success is dependent on responding to the previous relevant dimension and on ignoring the new, irrelevant dimension.

At the 'intra-dimensional shift' (IDS) stage, the subjects continued to respond to the novel exemplars within the previous relevant dimension and to ignore the irrelevant dimension. Following its reversal, the 'extra-dimensional shift' (EDS) and its reversal were pre- 
sented, again using novel exemplars of each stimulus dimension. At this stage, the subject had to learn that the previously irrelevant dimension was now relevant and the previously relevant dimension was now irrelevant in order to correspond correctly.

The EDS stage is equivalent to a category shift in the WCST and has been shown to be sensitive to frontal lobe (Owen et al. 1991) and basal ganglia dysfunction (Downes et al. 1989; Lawrence et al. 1996). The main measures of performance were the stage of the task successfully attained and errors made.

Spatial Working Memory Task. This CANTAB test of spatial working memory is a self-ordered searching task which requires the subject to search through a spatial array of colored boxes for "blue tokens" and has been shown to be sensitive to effects of frontal and temporal lobe lesions (Owen et al. 1990, 1995b, 1996b). To find a token, subjects need only to touch a box on the screen.

There was a training phase of three boxes and increasing to four, six, and eight boxes, respectively. Errors were scored according to the number of times the subject searched in a box for a token where one had already been found (a 'between search' error). 'Within search' errors (returning to a box already opened, and shown to be empty earlier in the same trial) were also measured. In addition, a strategy score was computed, that has been shown to be sensitive to frontal lobe dysfunction (Owen et al. 1990). The extent to which a strategy was used was established from a number of search sequences starting with a novel box for the six- and eight-box problems. A high score represented poor use of strategy and vice versa (range 1-37). The best score of 1 was obtained when the same box was used to initiate each search sequence at the more difficult six and eight box problems.

One-Touch Tower of London Task. To familiarize subjects with the task, a modified version of the Tower of London spatial planning task dependent on prefrontal cortical functioning (Shallice 1982) was used on a training session (Owen et al. 1995a). Two sets of colored balls hanging in stockings were presented on the screen. Subjects had to arrange the bottom pattern of balls according to specified rules to match the top pattern of arrangement by touching a ball and moving it to the wanted location. In the one-touch Tower of London task, subjects were again shown two arrangements of colored balls hanging in stockings and asked to determine, using the previously defined rules and without actually moving the balls, the minimum number of moves it would take to make the bottom arrangement match the goal arrangement. The bottom of the screen presented with five boxes labeled 1-5 and subjects had to touch the number which corresponded to the minimum number of moves required to solve the problem. Accuracy and latency measures were recorded.
Visuospatial Strategy Task. The visuospatial strategy task has been shown to be sensitive to impairments in patients with Parkinson's disease or frontal lobe injury (Owen et al. 1995a). Four symmetrically arranged red boxes were presented on the screen. Subjects were instructed to produce as many novel sequences as possible out of twenty-four by touching all four boxes in different orders once on each try (Owen et al. 1995a; Iddon et al. 1998). After the first twenty-four attempts, the subject moved on to the training phase where again four red boxes appeared on screen, but this time one box was surrounded by a white border. Six sequences were then generated starting with the highlighted box. This exercise was repeated with three other boxes that were highlighted. After this stage, the subjects again were presented with four unhighlighted red boxes and asked to generate 24 possible sequences improving upon their initial number of novel sequences generated.

The number of sequences generated out of 24 for both stages 1 and 2 were measured. Strategic performance was assessed by calculating the number of blocks of five or more starting with the same box in a row. The range of possible scores were thus between 0 and 4 . Perseveration was defined as immediate repetition of an entire sequence of four responses. The span score was the number of correct sequences occurring from the first sequence without an error (repetition of a previous sequence).

\section{Data Analysis}

All data were analysed using the Statistical Package for the Social Sciences (SPSS) version 7 in Windows 95 on a PC computer. One and two-way analyses of variance were performed, with subject group as the betweensubjects factor, and stage or difficulty level as the within subjects factor. Where appropriate, data were transformed prior to analysis to reduce distribution skewness and ensure homogeneity of variance (Howell 1997). When the parametric statistical test assumptions were not met i.e., values were recorded as 0 or 1 , a nonparametric Kruskal-Wallis one-way analysis of variance using ranks was conducted. For each test, planned between group pairwise contrast analyses were conducted and tested by the t-statistic. Nonparametric contrasts were carried out by performing separate MannWhitney U analyses.

Two comparisons were made. The drug groups were combined and compared to the controls and the drug groups were compared to each other. All multiple comparisons were Bonferroni corrected. It is possible that some of the variation of the differences in test performance among the groups was due to the level of education attained, as there were small differences between the drug abuser and control groups. Hence, in a parallel analysis, level of education was included as a covariate. 
Table 2. Mean Test Performance and Standard Deviations (SD) for the Heroin and Amphetamine Abuse Groups and Controls

\begin{tabular}{|c|c|c|c|}
\hline & Heroin Abusers & Amphetamine Abusers & Control Subjects \\
\hline \multicolumn{4}{|l|}{ Verbal fluency: } \\
\hline Letter fluency (FAS) (/60s each letter) & $38.41(15.14)$ & $36.00(15.43)$ & $46.67(14.68)$ \\
\hline Category fluency (Animals) (/90s) & $23.55(5.61)$ & $21.83(5.76)$ & $26.52(8.10)$ \\
\hline \multicolumn{4}{|l|}{ Pattern recognition: } \\
\hline$(\%$ correct $/ 24)$ & $20.36(2.74)$ & $19.43(2.56)$ & $22.14(1.55)$ \\
\hline (latency) & $2865.18(1188)$ & $2497.91(1012)$ & $2072.91(528)$ \\
\hline \multicolumn{4}{|l|}{ Spatial recognition: } \\
\hline$(\%$ correct $/ 20)$ & $15.09(3.18)$ & $15.74(2.47)$ & $17.32(1.76)$ \\
\hline (latency) & $2924.55(1211)$ & $2477.57(902)$ & $2271.82(760)$ \\
\hline \multicolumn{4}{|l|}{ Spatial working memory: } \\
\hline Total 'between search' errors & $32.36(23.65)$ & $28.57(20.50)$ & $16.64(11.34)$ \\
\hline Total 'within search' errors & $5.50(10.94)$ & $2.36(4.70)$ & $1.64(2.08)$ \\
\hline Strategy score & $34.14(4.52)$ & $29.77(6.02)$ & $31.77(4.51)$ \\
\hline \multicolumn{4}{|l|}{ One-touch Tower of London: } \\
\hline Perfect solutions (\% correct/12) & $61.76(19.94)$ & $64.66(24.33)$ & $81.05(11.75)$ \\
\hline \multicolumn{4}{|c|}{ Sequence Generation Task (24 possible sequences) } \\
\hline \multicolumn{4}{|l|}{ Sequence Generation: } \\
\hline Stage 1 & $16.95(1.95)$ & $15.83(3.17)$ & $17.32(2.61)$ \\
\hline Stage 2 & $17.52(2.52)$ & $18.00(3.50)$ & $20.73(2.91)$ \\
\hline \multicolumn{4}{|l|}{ Strategy: } \\
\hline Stage 1 & $0.19(0.40)$ & $0.12(0.33)$ & $0.55(1.06)$ \\
\hline Stage 2 & $0.90(1.30)$ & $1.12(1.54)$ & $2.59(1.71)$ \\
\hline \multicolumn{4}{|l|}{ Perseveration: } \\
\hline Stage 1 & $0.71(0.78)$ & $0.24(0.44)$ & $0.45(0.51)$ \\
\hline Stage 2 & $0.76(0.77)$ & $0.47(0.80)$ & $0.45(0.67)$ \\
\hline Span score & $7.48(4.58)$ & $10.29(4.84)$ & $8.64(4.32)$ \\
\hline
\end{tabular}

Only when a significant group effect was removed as a consequence of this analysis of covariance is it noted in the text.

Pearson's product moment and Spearman rank order correlational analyses were also conducted to assess the influence of such factors as drug abuse duration: a level of $p<.01$ was used to assess significance because of the large number of correlations computed. The index of variability shown in all cases was the standard deviation. For the attentional set-shifting task, data in the form of frequencies (the number of subjects passing or failing at each stage of the test) were case in contingency tables and analysed using likelihood ratio analysis (Robbins 1977). This method is useful for analysing data with small cell frequencies and is distributed as $\chi^{2}$.

\section{RESULTS}

\section{Verbal Fluency}

The chronic drug abuse groups both generated fewer words than the controls. Following a square-root transformation, this effect was significant in the case of the letter fluency (FAS) component $[\mathrm{F}(2,63)=3.37, p<.05]$ but not in the case of the semantic ('animals') component of the task. However, the significant group difference did not survive a supplementary analysis of covariance with level of education attained as a covariate.

\section{Pattern and Spatial Recognition}

Mean percentage correct scores for pattern and spatial recognition memory tests are shown in Table 2 . There was a significant difference among the three groups for both pattern recognition $[\mathrm{F}(2,64)=7.64, p<.01]$ and spatial recognition $[\mathrm{F}(2,64)=4.50, p<.05]$. The combined drug abuse group was impaired on pattern and spatial recognition memory compared to their control subjects [ $\mathrm{t}(64)=3.67, p<.01]$ and $[\mathrm{t}(64)=2.89, p<.01]$, but not impaired in relation to each other on either measure. However, the significant group effect for spatial recognition (though not pattern recognition) failed to survive analysis of covariance with level of education as a covariate.

Mean response latencies (see Table 2) were log transformed before analysis. There was a significant difference between the groups for pattern $[\mathrm{F}(2,64)=4.59, p<$ $.05]$ but not spatial $[\mathrm{F}(2,64)=2.71, p<.05]$ recognition. The combined drug abuse group was significantly impaired compared to their controls $[\mathrm{t}(64)=2.65, p<$ .025] but there was no difference in performance between the amphetamine and heroin abusers.

\section{Attentional Set-Shifting Task}

Figure 1a shows the cumulative percentage of subjects succeeding at each stage of the task. As can be seen from this figure, $91 \%$ of the control subjects compared 


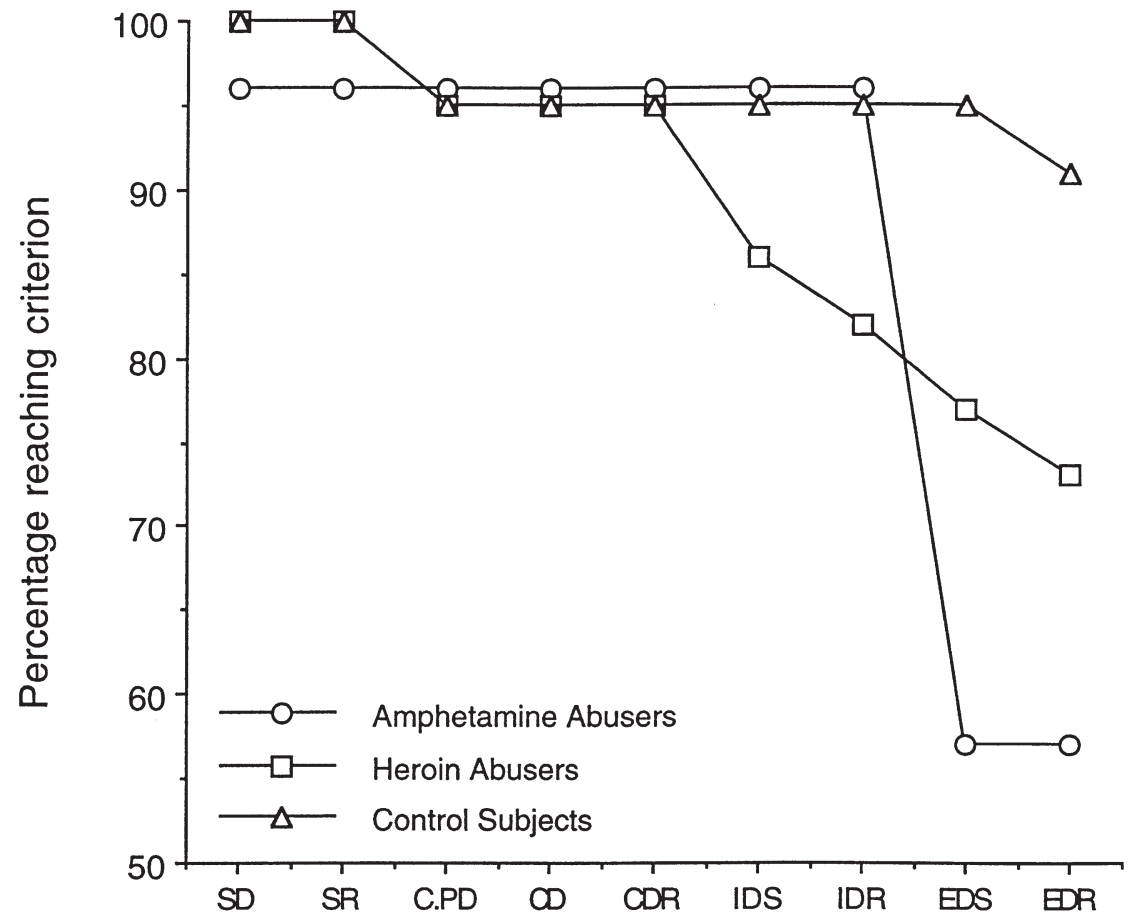

Figure 1. Proportion of subjects reaching the learning criterion (within 50 trials) at each stage of the visual discrimination/attentional set-shifting paradigm for the chronic amphetamine abusers, chronic heroin abusers, and IQ- and age-matched controls. SD, simple discrimination learning; SR, simple reversal learning; $C P D$, compound discrimination learning, separated elements; CD, compound discrimination learning; CDR, compound discrimination reversal; IDS, intra-dimensional shift; IDR, intra-dimensional reversal; EDS, extra-dimensional shift; EDR, extra-dimensional reversal.

\section{Stage}

with $73 \%$ of the heroin abuse group and $57 \%$ of the amphetamine abusers successfully completed all stages of the task. Overall, there was a significant difference among the groups in completing the task $\left[\chi_{2}{ }^{2}=7.30, p<\right.$ .05]. The difference between the combined drug group and the controls just reached significance $\left[\chi_{1}^{2}=5.60\right.$, $p<.05$ ] with $35.6 \%(16 / 45)$ of the drug abusers and only $9.1 \%(2 / 22)$ of the control subjects failing this task. No difference emerged between the amphetamine and heroin abusers for this gross index of performance.

However, performance at the EDS stage effectively differentiated between the groups for those subjects able to attempt that stage $\left[\chi_{2}{ }^{2}=17.18, p<.05\right]$. The control subjects performed significantly better than the combined drug group $\left[\chi_{1}{ }^{2}=9.23, p<.05\right]$ and significantly fewer of the amphetamine compared with the heroin abusers successfully passed this stage $\left[\chi_{1}{ }^{2}=\right.$ 7.95, $p<.01]$.

An additional two-way analysis of variance was performed to directly compare errors made at the IDS and EDS stages for the three groups using square-root transformed scores (see Figure 2). There was a significant group $\times$ stage interaction $[\mathrm{F}(2,64)=17.0, p<.001]$. The EDS and IDS stages were then analysed separately to determine simple main effects of group. For the EDS stage, there was also a significant difference between the combined drug group and the controls $[t(64)=3.32$, $p<.01]$ with the controls making fewer errors. Impor- tantly, the amphetamine abusers made significantly more errors than the heroin abusers [ $\mathrm{t}(64)=3.56, p<.01]$.

A parallel analysis was performed for errors made at the IDS stage. Again, there were differences among the three groups $[\mathrm{F}(2,64)=4.32, p<.05]$ which reflected overall differences between the heroin abusers and the other groups $[\mathrm{t}(64)=2.38, p<.02]$. A direct contrast applied between the amphetamine and heroin abuse groups confirmed that the latter performed significantly worse $[\mathrm{t}(64)=2.60, p<.01]$.

Additional post-hoc analyses comparing IDS and EDS errors in each group separately confirmed that there were differences among the three groups $[\mathrm{F}(2,64)=$ $4.32, p<.05]$ which reflected overall differences between the heroin abusers and the other groups $[\mathrm{t}(64)=$ $2.38, p<.02]$. A direct contrast applied between the amphetamine and heroin abuse groups confirmed that the latter performed significantly worse $[\mathrm{t}(64)=2.60, p<$ .01]. Finally, additional post-hoc analyses comparing IDS and EDS errors in each group separately confirmed that there were significant IDS-EDS differences for the controls and the amphetamine abusers, but not for the heroin abusers.

In summary, whereas the amphetamine abuse group made significantly more errors than controls or heroin abusers at the EDS stage, by contrast, the heroin abuse group made more errors at the IDS stage than the amphetamine or control subjects. Additionally, only the 


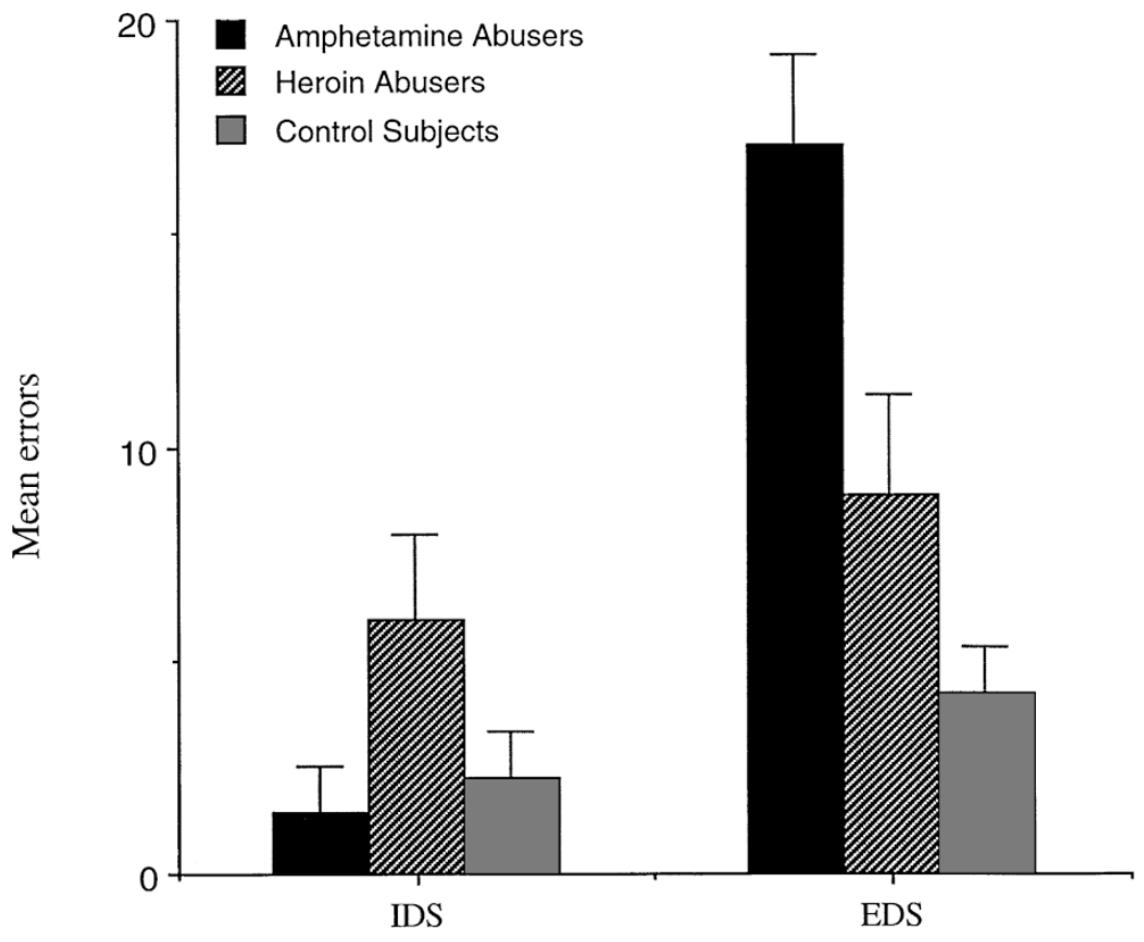

Stage
Figure 2. Mean (+ sem) of numbers of errors made at the intra-dimensional shift (IDS) and extra-dimensional shift (EDS) stages of the three groups. control and amphetamine groups showed the normal advantage for IDS over EDS performance.

\section{Spatial Working Memory}

Accuracy of Performance. For total 'between search' errors, following a square-root transformation, there was a significant difference between the groups $[\mathrm{F}(2,64)=$ $4.16, p<.05$ ] which reflected the control subjects making fewer errors than the combined drug group $[\mathrm{t}(64)=$ $2.81, p<.01]$. However, this difference did not survive analysis of covariance with level of education as a covariate. There was no difference in performance between the amphetamine and heroin abusers. For 'within search' errors, a Kruskal-Wallis comparison between the three groups revealed no difference on this measure (see Table 2).

Strategy Score. The mean strategy scores are presented in Table 2 . There was a significant difference between the groups $[\mathrm{F}(2,63)=4.25, p<.05]$. The heroin abuse group made less use of an efficient search strategy $[\mathrm{t}(63)=2.91, p<.01]$ than the amphetamine abusers. There was no difference between the combined drug group and the control subjects.

In summary, both groups made more errors than controls on this task, but the effect was in part linked to reduced education in the drug abuse groups. Only the heroin abusers exhibited an impaired strategy score.

\section{One-Touch Tower of London}

Accuracy of Performance. For 'perfect solutions', there was a significant effect of group $[\mathrm{F}(2,55)=5.63, p<$ $.01]$ which was due to the combined drug abuse groups being significantly less accurate than the controls, but not different from one another (see Table 2). A squareroot transformation of the number of choices to solution measure followed by analysis of variance conducted across the five difficulty conditions, revealed a significant interaction between group and difficulty levels $[F(8,240)=2.10, p<.05]$. The difference in performance between the combined drug abuse group and the control subjects approached significance at four-move $[\mathrm{t}(60)=2.10, p=.04$, but did not reach significance following Bonferroni connection] and five-move problems $[\mathrm{t}(60)=1.81, p=.076]$ (mean values for the more difficult levels are presented in Table 2). There was no difference in performance between the amphetamine and heroin abusers at any level of difficulty.

Latency. Three latency measures were also calculated: 1) the overall latency (latency to the correct solution regardless of the number of attempts); 2) the la- 
tency to the first response, regardless of whether that response was correct; and 3) latency to the first correct response (perfect solutions).

Logarithmically transformed latencies to the first response and to first correct response revealed only highly significant effects of difficulty level $[\mathrm{F}(4,228)=$ $116.29, p<.01]$ and $[\mathrm{F}(4,188)=140.00, p<.01]$ with latencies for all groups increasing as difficulty level increased. However, a difference in performance between the groups for both latencies to the first response and first correct response approached significance $[\mathrm{F}(2,57)=$ $3.11, p=.052]$ and $[\mathrm{F}(2,47)=3.01, p=.059]$. There were no group $\times$ difficulty level interactions for either measure. Overall latency also showed a significant effect of difficulty level $[\mathrm{F}(4,228)=142.4, p<.01]$ and there was a significant effect of group $[\mathrm{F}(2,57)=5.29, p<.01]$. The combined drug group performed significantly slower than the control subjects. There were however, no latency differences between the heroin and amphetamine abusers and no interaction between group and difficulty level emerged.

In summary, the drug abuse groups both performed less accurately on this planning task and were slower to respond according to certain latency measures. However, there were no differences between the amphetamine and heroin abuse groups and no informative interactions were shown with difficulty level.

\section{Visuospatial Strategy Task}

Sequences Generated. The mean numbers of sequences generated by the two drug groups over stages 1 and 2 (out of a possible 24) are presented in Table 2. There was a significant group effect $[\mathrm{F}(2,58)=5.32, p<$ $.01]$ and group $\times$ session interaction $[\mathrm{F}(2,58)=3.72, p<$ .05]. Analysis of the simple main effects of groups at each stage demonstrated that there was a significant difference in performance at stage 2 between the groups $[\mathrm{F}(2,58)=7.19, p<.01]$ but not at stage 1 . The combined drug abuse group performed significantly worse than the controls [t(58) $=3.73, p<.01]$ at stage 2 , but there were no differences in performance between the heroin and amphetamine abusers at this stage of the task.

However, analysis of the simple main effect of stage across each group showed that the heroin abusers' performance did not differ across stages 1 and 2 whereas both the amphetamine abuse and control groups improved significantly at stage $2[\mathrm{t}(17)=2.16, p<.05]$ and $[\mathrm{t}(21)=5.88, p<.01]$.

In summary, both of the abuser groups were equivalently impaired on overall performance of this task, but the heroin group showed significantly less improvement following training.

Strategy Learning. A Kruskal-Wallis comparison revealed that the three groups did not perform signifi- cantly differently at stage 1 , but did at stage $2\left(\chi_{2}^{2}=\right.$ $11.48, p<.01)$ with the control subjects performing better than the combined heroin and amphetamine abuse group $\left(\chi_{1}{ }^{2}=11.38, p<.01\right)$. There was no difference in performance between the drug abuse groups (see Table 2).

Perseveration Score. There was a near significant difference among the groups $[\mathrm{F}(2,57)=3.05, p<.055]$ which, from inspection of the means in Table 2, probably arose from a tendency for the heroin abusers to exhibit higher perseveration scores. In fact, a contrast applied between this group and the combined control and amphetamine abuser groups revealed a significant difference $[\mathrm{t}(57)=2.43, p<.018]$.

Span Score. No difference between the groups emerged for this measure (see Table 2).

\section{Comparison of Subgroups in Chronic Amphetamine Abuse Group}

Further comparisons were made between the two subgroups within the amphetamine group: of subjects who also met the criteria for opiate dependence and were on concurrent methadone medication $(n=9)$ and the subgroup who did not meet criteria for opiate dependence, and who were not on methadone treatment $(n=14)$. The two groups were well matched for age and IQ, showing no significant differences. The results of the analyses for the main neuropsychological test variables, together with mean (SD) values, are shown in Table 3. As can be seen, in general, the results for the 'amphetamine alone' subgroup are very similar to those for the larger group described above. There were also no significant differences between the two subgroups.

\section{Correlational Analysis}

Pearson Product Moment correlation coefficient or Spearman rank order correlation coefficients were calculated between cognitive test scores for most tests, level of education, age and overall drug abuse duration, amphetamine abuse duration and heroin abuse duration. No correlations attained significance at the stringent $p<.01$ criterion.

\section{DISCUSSION}

The present study has compared the neuropsychological profile of two groups of chronic drug abusers, predominantly or heroin or amphetamine, on a novel computerised neuropsychological battery, including some tests or variants from CANTAB, which has been validated extensively in patients with cortical damage, neuropsychiatric disorders, and neurodegenerative disease, as well as in normal volunteers in neuroimaging 
Table 3. Cognitive Test Performance Comparisons in Sub-Groups with Chronic Amphetamine Abuse with or without Additional Opiate Dependence

\begin{tabular}{|c|c|c|c|c|c|c|}
\hline \multirow[b]{2}{*}{ Test } & \multicolumn{3}{|c|}{ Group Effect } & \multicolumn{3}{|c|}{ Mean (SD) } \\
\hline & $\mathbf{F}$ & DF & $\mathbf{P}$ & $\mathrm{C}$ & AMP & AMP/OP \\
\hline Letter Fluency (FAS) & 3.79 & $(2,41)$ & $<.05$ & $46.67(14.68)$ & $32.79(10.29)^{* *}$ & $41.00(20.89)$ \\
\hline Category Fluency (Animals) & 3.09 & $(2,41)$ & $>.05$ & $26.52(8.10)$ & $20.57(6.16)$ & $23.78(4.74)$ \\
\hline Pattern Recognition & 9.31 & $(2,42)$ & $<.001$ & $22.14(1.55)$ & $19.71(2.43)^{* *}$ & $19.00(2.83)+$ \\
\hline Spatial Recognition & 2.96 & $(2,42)$ & $>.05$ & $17.32(1.76)$ & $15.79(2.33)$ & $15.67(2.83)$ \\
\hline \multicolumn{7}{|l|}{ Attentional set-shifting } \\
\hline EDS Errors & 17.69 & $(2,42)$ & $<.001$ & $4.23(5.05)$ & $19.00(10.11)^{* *}$ & $14.11(10.17) \dagger$ \\
\hline IDS Errors & 1.19 & $(2,42)$ & $>.05$ & $2.23(5.12)$ & $2.07(6.62)$ & $0.44(0.73)$ \\
\hline SWM Between Search Errors & 2.73 & $(2,42)$ & $>.05$ & $16.64(11.34)$ & $31.86(23.13)$ & $23.44(15.40)$ \\
\hline SWM Strategy Score & 0.44 & $(2,42)$ & $>.05$ & $31.77(4.51)$ & $30.36(6.38)$ & $30.11(5.78)$ \\
\hline TOL Perfect Solutions (\% correct) & 3.40 & $(2,34)$ & $<.05$ & 81.05 (11.75) & $63.93(27.26)^{*}$ & $66.54(16.76)$ \\
\hline \multicolumn{7}{|l|}{ VST Sequences Generated } \\
\hline (averaged across stages 1 and 2 ) & 3.48 & $(2,37)$ & $<.05$ & $19.02(2.41)$ & $16.88(2.79)^{*}$ & $17.00(2.28)$ \\
\hline
\end{tabular}

paradigms. There were qualitative differences in cognitive functioning between the chronic amphetamine and heroin groups, notably on a test of visual discrimination learning and shifting, and in some aspects of visuospatial memory function. Both groups were profoundly impaired on a test of visual pattern recognition memory which has been shown to be sensitive to temporal lobe lesions (Owen et al. 1995b). This deficit was a selective one, in that it survived analyses taking account of years of education, and it was also readily apparent in the relatively 'pure' subgroup of chronic amphetamine abusers who did not also abuse opiates.

This is a unique study, as there are relatively few published data comparing cognitive deficits in clinical populations of chronic stimulant and opiate users. In the United Kingdom some treatment centers do prescribe amphetamines and almost half the amphetamine sample in this study were taking daily prescribed amphetamines of known purity. The chronic amphetamine and chronic heroin groups were distinguished on the basis of DSM-IV criteria for amphetamine and opiate dependence, and despite some of the amphetamine group also fulfilling criteria for opiate dependence, significant qualitative differences in cognitive functioning were found between the two groups, thus helping to validate this approach. When the opiate dependent abusers were excluded from the chronic amphetamine group, the same qualitative pattern of significant differences was found in neuropsychological test variables (Table 3). Therefore, the effects shown in the chronic amphetamine group could not be attributed to opiate dependence or to acute effects of methadone.
One caveat in interpreting the findings is that the monitoring of drug-taking behavior close to the test session was not confirmed, for example, by urine or plasma analyses, so that it is just possible, despite our clinical screening procedure, that some of the subjects in the chronic abuse group were still performing while under the effects of the abused drug, or alternatively, methadone. There is comparative information available on the effects of acute methylphenidate in volunteers for this battery (Elliott et al. 1997) — which shows for the most part that this drug often enhances rather than impairs performance, at least for the non-drug abusing normal population.

A related methodological problem was the lack of objective assessment of the absence of abstinence symptoms for amphetamine: these were monitored instead by the clinician caring for these individuals. However, the different patterns of cognitive deficit exhibited in the two groups effectively argue against a general withdrawal-induced malaise or motivational impairment producing non-specific disruptions of performance. Other possible problems of interpretation related to coincidental differences between control individuals and drug abusers, such as anti-social personality and level of education attained. The latter influence was ruled out by means of an analysis of covariance for the main neuropsychological differences reported here. Although the possible influence of anti-social personality was not explicitly controlled, a parallel study of a large group of patients with antisocial and/or borderline personality disorder, the majority exhibiting varying degrees of drug misuse, showed a somewhat different profile of 
impairment, which for example, did not include impairments in visual recognition memory (Bazanis et al. unpublished observations).

The most striking result in the present study was the specific impairment in EDS performance in the amphetamine group, with sparing of related tests from the same suite of discrimination tests, including the IDS. This deficit in set-shifting was even clearer in the subgroup of amphetamine abusers who were not also on methadone. The EDS is formally equivalent to the category shift in the Wisconsin Card sorting Test. The EDS stage deficit has also been observed in patients with basal ganglia disorders, such as Huntington's disease (Lawrence et al. 1996) and in neurosurgical patients with frontal, but not temporal lobe excision (Owen et al. 1991). By contrast, the heroin group were less impaired on the EDS stage, although they were more impaired at earlier stages of the test, including the IDS, which involves abstraction and learning set.

The failure of subjects in the heroin group to show efficient IDS performance (as well as a general tendency to make more errors at even earlier stages; data not shown), may be taken to indicate a deficit in 'tuning in' to the relevant dimension as a general consequence of impaired reinforcement learning. This failure to 'tune in' may explain the relatively weaker nature of their EDS deficit which depends on the need to shift from a selectively attended perceptual dimension (such as 'shapes' or 'lines' in our test). Performance on the IDS is not generally impaired by frontal or basal ganglia dysfunction (Dias et al. 1996; Owen et al. 1991). Furthermore, it appears that differences in education between the groups were not responsible for the deficits; the significant ID/ED performance differences were preserved following analysis of covariance with level of education attained as the covariate.

The findings for the ID/ED task extend previous observations made on cocaine (crack) abusers and other polydrug abuse groups. Rosselli and Ardila (1996) however, found that neither perseverative nor non-perseverative errors effectively distinguished their cocaine-dependent and polydrug abusing groups. O'Malley et al. (1992) found that their cocaine abusing group performed more poorly on the Halstead Category Test, which assesses abstracting ability. Press (1983) found that the Luria-Nebraska Neuropsychological Battery failed to differentiate deficits caused by cocaine use from those of other psychoactive drugs. It is, of course, possible that although both drugs are in the stimulant class, chronic amphetamine abuse (as studied here) produces different effects from those of chronic cocaine.

Impairments shown by the chronic amphetamine group on other tests sensitive to fronto-striatal dysfunction, such as verbal fluency (FAS), the Tower of London test of planning, spatial recognition, and the selfordered spatial working memory and visuospatial strategy tasks (see e.g., Owen et al. 1990, 1995b; Iddon et al. 1998 and unpublished observations) were generally less convincing. In some cases (e.g., spatial recognition, verbal fluency) they did not survive analysis which took into account years of education; in others (e.g., Tower of London test of planning) they were not selective to the amphetamine group, being also evident in the chronic heroin abusers. On some measures, deficits in the latter group were actually more prominent. For example, unlike the amphetamine group, the chronic heroin abusers showed impaired strategy on the spatial working memory task (despite overall memory performance being comparable to that of the amphetamine abusers) and also showed no sign of improvement between stages 1 and 2 of the sequence generation task, even from levels of performance comparable to those of controls on stage 1. Thus, the greater deficits on the EDS in the amphetamine group were evidently not a result of greater overall intellectual deficit, as the heroin group showed greater impairments in other domains. These impairments included executive dysfunction in the visuospatial domain, as well as the general learning deficits seen on the visual discrimination tasks in the ID/ED paradigm. The former results may stand in contrast to the general conclusion of previous studies (see Introduction) that heroin abusers do not exhibit typical frontal-executive deficits.

Some of our previous findings support the hypothesis of frontal-executive dysfunction in chronic drug abusers (Rogers et al. 1999b). A group of amphetamine abusers, including many of those also tested here, were impaired in several aspects of performance in a decision-making task, including the speed and quality of decision-making. In contrast, a parallel group of heroin abusers, also including many of those tested here, were only significantly impaired in their speed of decisionmaking. The pattern of deficits in the amphetamine group matched those observed in a group of patients with ventromedial, but not dorso-lateral or medial prefrontal damage. They also matched data obtained in normal volunteers subjected to tryptophan depletion procedure. Together, these results are consistent with evidence that amphetamine abuse is associated with loss of 5-HT from the orbitofrontal cortex (Wilson et al. 1996a). It is possible that the reductions in dopamine transporter activity also seen in that group (c.f. Wilson et al. 1996b) reflect some of the other impairments seen in this study, for example given significant relationships between striatal dopamine receptor binding and some of the tests (e.g. Tower of London, visuospatial strategy task) and in the present study (Lawrence et al. 1998).

One implication of these findings is that amphetamine and heroin abuse can lead to cognitive deficits through lasting effects on cortico-striatal circuitry. This conclusion is supported by the work of Volkow et al. 
(1997) on the response to stimulants such as methylphenidate in cocaine abusers, as well as by other work suggesting significant cerebral hypoperfusion in the periventricular, frontal and other neocortical regions following cocaine abuse (e.g., Strickland et al. 1993).

The deficits described here may add to the burden of rehabilitating both amphetamine and heroin abusers. Thus, where there is evidence of specific cognitive impairments in shifting behavior or in decision-making (Rogers et al. 1999b) such deficits may exacerbate those patterns of behavior leading to further drug abuse, perhaps as ancillary neurotoxic consequences of drug abuse. There is an extensive literature on neurotoxic effects of amphetamine and related drugs in experimental animals, although it can be questioned to what extent such toxicity might be expressed in human drug abusers (Ricaurte et al. 1984; Ryan et al. 1990; Melega et al. 1996). Nevertheless, recent studies (e.g., Robinson and Kolb 1997, 1999) are now demonstrating apparently long-lasting changes in dendritic branching in brain regions such as the nucleus accumbens and parietal and prefrontal cortex, of repeated injections of single doses of morphine or amphetamine in ranges relevant to reinforcing effects. Matching such patterns of anatomical connectivity to similar brain changes in human drug abusers and associated, possibly drug-specific, changes in cognitive function is a target for future research.

\section{ACKNOWLEDGMENTS}

This work was supported by a Programme Grant from the Wellcome Trust. The work was completed within the MRC Co-operative for Brain, Behaviour and Neuropsychiatry. We thank T. Carthy and J. Hunter for assistance and RD Rogers for discussion.

\section{REFERENCES}

Alexander GE, DeLong MR, Strick PL (1986): Parallel organization of functionally segregated circuits linking basal ganglia and cortex. Rev Neurosci 9:357-381

Ardila A, Rosselli M, Strumwasser S (1991): Neuropsychological deficits in chronic cocaine abusers. Intl J Neurosci 57:73-79

Baker SC, Rogers RD, Owen AM, Frith CD, Dolan RJ, Frackowiak RSJ, Robbins TW (1996): Neural systems engaged by planning: A PET study of the tower of London task. Neuropsychologia 34:515-526

Benton AL (1968): Differential behavioural effects of frontal lobe disease. Neuropsychologia 6:53-60

Bruhn P, Maage N (1975): Intellectual and neuropsychological functions in young men with heavy and long-term patterns of drug abuse. Am J Psychiatry 132:397-401
Carlin AS, O'Malley S (1996): Neuropsychological consequences of drug abuse. In Grant I, Adams KM (eds), Neuropsychological Assessment of Neuropsychiatric Disorders. New York, Oxford University Press, pp 486-503

Dias R, Roberts A, Robbins TW (1996): Dissociation in prefrontal cortex of affective and attentional shifts. Nature 380:69-72

Task Force of American Psychiatric Association (1994): Diagnostic and Statistical Manual of Mental Disorders-4th Edition. Washington, DC, American Psychiatric Association

Downes JJ, Roberts AC, Sahakian BJ, Evenden JL, Morris RG, Robbins TW (1989): Impaired extra-dimensional shift performance in medicated and unmedicated Parkinson's disease: Evidence for a specific attentional dysfunction. Neuropsychologia 27(11/12):1329-1343

Elliott R, Sahakian BJ, Matthews K, Bannerjea A, Rimmer J, Robbins TW (1997): Effects of methylphenidate on spatial working memory and planning in healthy young adults. Psychopharmacology 213:1-11

Elliott R, McKenna PJ, Robbins TW, Sahakian BJ (1998): Specific neuropsychological deficits in schizophrenic patients with preserved intellectual function. Cogn Neuropsychiatry 3:45-70

Grant DA, Berg EA (1948): A behavioural analysis of degree of reinforcement and ease of shifting to new responses in a Weigl-type card-sorting problem. J Exp Psychol 38:404-411

Grant I, Adams KM, Carlin AS, Rennick PM, Judd LL, Schooff K (1978): Collaborative neuropsychological study of polydrug users. Arch Gen Psychiatry 35:10631074

Haarhoff G, London M (1995): A comparative study of injecting opiate and amphetamine users in a rural area. Addict Res 3(1):33-38

Hill SY, Mikhael MA (1979): Computerised transaxial tomographic and neuropsychological evaluations in chronic alcoholics and heroin abusers. Am J Psychiatry 136:598602

Howell DC (1997): Statistical Methods for Psychology. Belmont, CA, Duxbury Press

Iddon JL, McKenna PJ, Sahakian BJ, Robbins TW (1998): Impaired generation and use of strategy in schizophrenia: Evidence from visuospatial and verbal tasks. Psychol Med 28:1049-1062

Joyce N, Meador-Woodruff JH (1997): Linking the family of $\mathrm{D}_{2}$ receptors to neuronal circuits in human brain: Insights into schizophrenia. Neuropsychopharmacology 16:375-384

Kalivas PW, Sorg BA (1997): Animal models of psychosis reveal involvement of hippocampal-corticostriatal-mesencephalic circuitry. In Beninger RJ, Palomo T, Archer T (eds), Dopamine Disease States. Madrid, Spain, Editorial CYM

Koob GF, LeMoal M (1997): Drug abuse: hedonic homeostatic dysregulation. Science 278:52-58

Lange K, Robbins TW, Marsden CD, James M, Owen A, Paul GM (1992): L-Dopa withdrawal selectively impairs performance in test of frontal lobe function in Parkinson's disease. Psychopharmacology 107:394-404 
Lawrence AD, Sahakian BJ, Hodges JR, Rosser AE, Lange KW, Robbins TW (1996): Executive and mnemonic functions in early Huntington's disease. Brain 119:15971615.

Lawrence AD, Weeks RA, Brooks DJ, Andrews TC, Watkins LHA, Harding AE, Robbins TW, Sahakian BJ (1998): The relationship between striatal dopamine receptor binding and cognitive performance in Huntington's disease. Brain 121:1343-1355

Mansour A, Khachaturian H, Lewis ME, Akil H, Watson SJ (1988): Anatomy of CNS opioid receptors. Trends Neurosci 11:308-314

Maruff P, Burns CB, Tyler P, Currie BJ, Currie J (1998): Neurological and cognitive abnormalities associated with chronic petrol sniffing. Brain 121:1903-1917

Melega WP, Quintana J, Raleigh MJ, Stout DB, Yu DC, Lin DP, Huang SC, Phelps ME (1996): 6-[ ${ }^{18}$ F]fluoro-[sca]1DOPA-PET studies show partial reversibility of long term effects of chronic amphetamine in monkeys. Synapse 22:63-69

Miller L (1985): Neuropsychological assessment of substance abusers: review and recommendations. J Subst Abuse Treat 2:5-17

Milner B (1963): Effects of different brain lesions on card sorting. Arch Neurol 9:90-100

Mittenberg W, Motta S (1993): Effects of cocaine abuse on memory and learning. Arch Clin Neuropsychol 8:477484

Nelson HE (1982): The National Adult Reading Test (NART). Windsor, NFER-Nelson

O'Malley S, Adams M, Heaton RK, Gawin FH (1992): Neuropsychological impairment in chronic cocaine abusers. Am J Drug Alcohol Abuse 18:131-144

Owen AM, Downes JJ, Sahakian BJ, Polkey CE, Robbins, TW (1990): Planning and spatial working memory following frontal lobe lesions in man. Neuropsychologia 28(10): 1021-1034

Owen AM, Roberts AC, Polkey CE, Sahakian BJ, Robbins TW (1991): Extra-dimensional versus intra-dimensional set shifting performance following frontal lobe excisions, temporal lobe excisions or amygdala-hippocampectomy in man. Neuropsychologia 29(10):993-1006

Owen AM, James M, Leigh PH, Summers BA, Marsden CD, Quinn NP, Lange KW, Robbins TW (1992): Fronto-striatal cognitive deficits at different stages of Parkinson's disease. Brain 115:1727-1751

Owen AM, Beksinska M, James M, Leigh PN, Summers BA, Marsden CD, Quinn NP, Sahakian BJ, Robbins TW (1993): Visuospatial memory deficits at different stages of Parkinson's disease. Neuropsychologia 3(7): 627-644

Owen AM, Sahakian BJ, Hodges JR, Summers BA, Polkey CE, Robbins TW (1995a): Dopamine-dependent frontrostriatal planning deficits in early Parkinson's disease. Neuropsychology 9(1):126-140

Owen AM, Sahakian BJ, Semple J, Polkey CE, Robbins TW (1995b): Visuo-spatial short-term recognition memory and learning after temporal lobe excision, frontal lobe excisions or amygdalo-hippocampectomy in man. Neuropsychologia 33(1):1-24

Owen AM, Evans AC, Petrides M (1996a): Evidence for a two-stage model of spatial working memory processing within the lateral frontal cortex: A Positron Emission Tomography study. Cerebr Cortex 6:31-38

Owen AM, Morris RG, Sahakian BJ, Polkey CE, Robbins TW (1996b): Double dissociation of memory and executive functions in working memory tasks following frontal lobe excision, temporal lobe excisions or amygdalo-hippocampectomy in man. Brain 119: 1597-1615

Parsons ES, Farr SD (1981): The neuropsychology of alcohol and drug abuse. In Filkskov SB, Boll TS (eds), Handbook of Clinical Neuropsychology. Vol. 1. New York, Wiley, pp 320-365

Press RJ (1983): The Neuropsychological Effects of Cocaine and Opiate Use. Ann Arbor, MI, University Microfilms International

Ricaurte GA, Schuster CR, Seiden LS (1984): Further evidence that amphetamines produce long-lasting dopamine neurochemical deficits by destroying dopamine nerve fibers. Brain Res 303:359-364

Robbins TW (1977): A critique of the methods available for the measurement of spontaneous locomotor activity. In Iversen L, Iversen S (eds), Handbook of Psychopharmacology. Vol. 7. New York, Plenum Press, pp 37-82

Robbins TW (1996): Dissociating executive functions of the prefrontal cortex. Philos Transact Royal Soc London 'B' 351:1463-1471

Robbins TW, James M, Owen AM, Sahakian BJ, McInnes L, Rabbitt P (1994): Cambridge neuropsychological test automated battery (CANTAB): A factor analytic study of a large sample of normal elderly volunteers. Dementia 5:266-281

Robbins TW, James M, Owen AM, Sahakian BJ, Lawrence AD, McInnes L, Rabbitt PMA (1998): A study of performance on tests from the CANTAB battery sensitive to frontal lobe dysfunction in a large sample of normal volunteers: Implications for theories of executive functioning and cognitive aging. J Intl Neuropsychol Soc 4:474-490

Robinson TE, Kolb B (1997): Persistent structural modifications in nucleus accumbens and prefrontal cortex neurons produced by previous experience with amphetamine. J Neurosci 17:8491-8497

Robinson TE, Kolb B (1999): Morphine alters the structure of neurons in the nucleus accumbens and neocortex of rats. Synapse 33:160-162

Rogers RD, Andrews TC, Grasby PM, Brooks D, Robbins TW (1999a): Contrasting cortical and sub-cortical PET activations produced by reversal learning and attentional-set shifting in humans. J Cogn Neurosci 12:142162

Rogers RD, Everitt BJ, Baldacchino A, Blackshaw AJ, Swainson R, Wynne K, Baker NB, Hunter J, Carthy T, Booker E, London M, Deakin JFW, Sahakian BJ, Robbins TW (1999b): Dissociable deficits in the decision-making cognition of chronic amphetamine abusers, opiate abusers, patients with focal damage to prefrontal cortex, and tryptophan-depleted normal volunteers: Evidence for monoaminergic mechanisms. Neuropsychopharmacology 20:322-339

Rosselli M, Ardila A (1996): Cognitive effects of cocaine and polydrug abuse. J Clin Exp Neuropsychol 18:122-135 
Rounsaville BJ (1982): Neuropsychological functioning in opiate addicts. Journal Nerv Mental Dis 170(4):209-216

Ryan LJ, Linder JC, Martone ME, Groves PM (1990): Histological and ultrastructural evidence that d-amphetamine causes degeneration in neostriatum and frontal cortex of rats. Brain Res 518:67-77

Sahakian BJ, Morris RG, Evenden JL, Heald A, Levy R, Philpot MP, Robbins TW (1988): A comparative study of visuo-spatial learning and memory in Alzheimertype dementia and Parkinson's disease. Brain 111:695718

Shallice T (1982): Specific impairment of planning. PhilosTransact Royal Soc 'B' London 298:199-209

Strickland TL, Mena I, Villanueva-Meyer J, Cummings K, Mehringer CM, Satz P, Myers H (1993): Cerebral perfusion and neuropsychological consequences of chronic cocaine use. J Neuropsychiatry 5:419-427

Volkow ND, Wang G-J, Fowler JS, Logan J, Gatley SJ, Hitzeman R, Chen AD, Dewey SL, Pappas N (1997): Decreased striatal dopaminergic responsiveness in detoxified cocaine-dependent subjects. Nature 386:830-833

Washton AM, Gold MS (1984): Chronic cocaine abuse: evidence and adverse effects on health and functioning. Psychiatric Ann 17:733-743

Wilson JM, Kalasinsky KS, Levey AI, Bergeron C, Reiber G, Anthony RM, Shchmunk GA, Shannak K, Haycock JW, Kish SJ (1996a): Striatal dopamine nerve terminal markers in human, chronic methamphetamine users. Nature Med 2(6):699-703

Wilson J, Levey A, Bergeron C, Kalasinsky K, Ang L, Peretti F, Adams VI, Smialek J, Anderson WR, Shannak K, Deck J, Niznik HB, Kish SJ (1996b): Striatal dopamine, dopamine transporter, and vesicular monoamine transporter in chronic cocaine users. Ann Neurol 40(3):428439

Wise RA, Bozarth MA (1984): Brain reward circuitry: four circuit elements "wired" in apparent series. Brain Res Bull 12:203-208 